\title{
Long-term efficacy and safety results of taliglucerase alfa through 5 years in adult treatment-naïve patients with Gaucher disease
}

\author{
Ari Zimran ${ }^{\mathrm{a}, *}$, Gloria Durán ${ }^{\mathrm{b}}$, Pilar Giraldo ${ }^{\mathrm{c}}$, Hanna Rosenbaum ${ }^{\mathrm{d}}$, Fiorina Giona ${ }^{\mathrm{e}}$, Milan Petakov ${ }^{\mathrm{f}}$, \\ Eduardo Terreros Muñoz ${ }^{g}$, Sergio Eduardo Solorio-Meza ${ }^{\text {h }}$, Peter A. Cooper ${ }^{\mathrm{i}}$, Sheeba Varughese ${ }^{\mathrm{i}}$, \\ Sari Alon ${ }^{\mathrm{j}}$, Raul Chertkoff ${ }^{\mathrm{j}}$ \\ ${ }^{a}$ Gaucher Clinic, Shaare Zedek Medical Center, Hebrew University and Hadassah Medical School, Jerusalem, Israel \\ b Pontificia Universidad Catolica de Chile, Santiago, Chile \\ c CIBERER, Hospital Universitario Miguel Servet, IIS Aragón, Zaragoza, Spain \\ d Rambam Medical Center, Haifa, Israel \\ e Sapienza University, Rome, Italy \\ ${ }^{\mathrm{f}}$ Clinical Center of Serbia, Clinic of Endocrinology, Diabetes and Metabolic Disease, Belgrade University Medical School, Belgrade, Serbia \\ g Centro Médico Nacional Siglo XXI, Servicio de Hematologia, Mexico City, Mexico \\ ${ }^{\text {h }}$ Hospital de Especialidades No1, Leon, Mexico

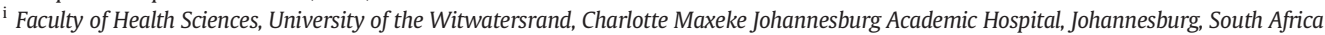 \\ ${ }^{j}$ Protalix BioTherapeutics, Carmiel, Israel
}

\section{A R T I C L E I N F O}

\section{Article history:}

Submitted 14 July 2016

Available online $\mathrm{xxxx}$

Editor: Mohandas Narla

\section{Keywords:}

Gaucher disease

Enzyme replacement therapy

Taliglucerase alfa

Splenomegaly

Hepatomegaly

Anemia

Thrombocytopenia

Chitotriosidase

Chemokine ( $\mathrm{C}-\mathrm{C}$ motif) ligand 18

\begin{abstract}
A B S T R A C T
Taliglucerase alfa, the first available plant cell-expressed recombinant therapeutic protein, is an enzyme replacement therapy approved for Gaucher disease (GD). PB-06-001, a pivotal phase 3, multicenter, randomized, double-blind, parallel-dose study investigated taliglucerase alfa 30 or $60 \mathrm{U} / \mathrm{kg}$ every other week through 9 months in treatment-naïve adults with GD; 30-month extension study PB-06-003 followed. Patients completing PB-06-001 and PB-06-003 could continue treatment in PB-06-007. Nineteen patients enrolled in PB-06-007 (30 U/kg, $\mathrm{n}=8 ; 60 \mathrm{U} / \mathrm{kg}, \mathrm{n}=9$; dose adjusted, $\mathrm{n}=2$ ); 17 completed 5 total years of treatment. In these 3 groups, respectively, taliglucerase alfa resulted in mean decreases in spleen volume $(-8.7,-6.9,-12.4$ multiples of normal), liver volume $(-0.6,-0.4,-0.5$ multiples of normal), chitotriosidase activity $(-83.1 \%,-93.4 \%,-87.9 \%)$, and chemokine ( $C-C$ motif) ligand 18 concentration $(-66.7 \%,-83.3 \%,-78.9 \%)$, as well as mean increases in hemoglobin concentration $(+2.1,+2.1,+1.8 \mathrm{mg} / \mathrm{dL})$ and platelet count $\left(+31,871,+106,800,+34,000 / \mathrm{mm}^{3}\right)$. The most common adverse events were nasopharyngitis and arthralgia. Most adverse events were mild/moderate; no serious adverse events were considered treatment-related. These results demonstrate continued improvement of disease parameters during 5 years of taliglucerase alfa therapy in 17 treatment-naive patients with no new safety concerns, extending the taliglucerase alfa clinical efficacy and safety dataset. This study was registered at www.clinicaltrials.gov as NCT01422187.
\end{abstract}

(c) 2016 The Authors. Published by Elsevier Inc. This is an open access article under the CC BY-NC-ND license (http://creativecommons.org/licenses/by-nc-nd/4.0/).
Abbreviations: AEs, adverse events; CCL18, chemokine (C-C motif) ligand 18; ECG, electrocardiogram; ERT, enzyme replacement therapy; FDA, US Food and Drug Administration; GD, Gaucher disease; IgG, immunoglobulin G; MN, multiples of normal, where normal spleen volume is $2 \mathrm{~mL} / \mathrm{kg}$ times body weight $(\mathrm{kg})$ and normal liver volume is $25 \mathrm{~mL} / \mathrm{kg}$ times body weight $(\mathrm{kg})$; QCSI, quantitative chemical shift imaging; SD, standard deviation; SE, standard error.

* Corresponding author at: Gaucher Clinic, Shaare Zedek Medical Center, 12 Bayit Street, P.O. Box 3235, Jerusalem 91031, Israel.

E-mail address: azimran@gmail.com (A. Zimran).

\section{Introduction}

The rare lysosomal storage disorder Gaucher disease (GD) is caused by mutations in the gene for the enzyme beta-glucocerebrosidase [1]. These mutations confer deficient activity of beta-glucocerebrosidase in macrophages and other cell types, resulting in lysosomal accumulation of glucocerebroside and leading to pathology that includes splenomegaly, hepatomegaly, anemia, and thrombocytopenia [1].

GD exists as a spectrum of disease with 3 major phenotypes: nonneuronopathic Type 1, acute neuronopathic Type 2, and chronic neuronopathic Type 3 [2]. The most common form is Type $1 \mathrm{GD}$; it can manifest at any age, from infancy through adulthood $[3,4]$. 
Enzyme replacement therapy (ERT) is the standard of care for patients with GD [5,6]. Taliglucerase alfa is an ERT approved for the treatment of adult patients with Type 1 GD in the United States, Israel, Brazil, Australia, Canada, Chile, and other countries, and is approved for the treatment of pediatric patients in the United States, Australia, Canada, Israel, Mexico, and other countries, and for hematologic manifestations of Type 3 GD in pediatric patients in Canada. Taliglucerase alfa is free of mammalian components, is produced in disposable bioreactors, and is the first plant cell-expressed recombinant therapeutic protein ever approved by the US Food and Drug Administration (FDA) for use in humans [7-9].

A phase 3, multicenter, randomized, double-blind, parallel-dose study (NCT00376168; Study PB-06-001) investigated treatment with taliglucerase alfa $30 \mathrm{U} / \mathrm{kg}$ or $60 \mathrm{U} / \mathrm{kg}$ through 9 months in treatment-naïve adult patients with GD [10]. In this initial pivotal study, patients demonstrated improvements in spleen and liver volume, hemoglobin concentration, platelet counts, and chitotriosidase activity with taliglucerase alfa $30 \mathrm{U} / \mathrm{kg}$ and $60 \mathrm{U} / \mathrm{kg}$ [10]. Treatmentrelated adverse events (AEs) were all mild or moderate in intensity, and no serious AEs were reported [10].

Patients who continued into extension study PB-06-003 (NCT00705939) demonstrated continued improvement in the disease parameters, and no new safety issues emerged $[11,12]$. Patients who completed Study PB-06-003 were eligible to continue treatment in an additional extension study, PB-06-007, for a total duration of treatment with taliglucerase alfa of up to 5 years. This report presents the efficacy and safety results for treatment-naïve adult patients with GD who received taliglucerase alfa at a dose of $30 \mathrm{U} / \mathrm{kg}$ or $60 \mathrm{U} / \mathrm{kg}$ for up to 5 total years of treatment.

\section{Materials and methods}

\subsection{Study design}

Patients were enrolled in extension study PB-06-007 (NCT01422187) after sequentially completing studies PB-06-001 and PB-06-003, representing 39 total months of treatment. Details of PB-06-001 and PB-06-003 have previously been reported [10-12]. In PB-06-007, patients continued to receive further treatment with taliglucerase alfa at the same dose, for an additional 21 months (total of 60 months), or until taliglucerase alfa became commercially available, at the discretion of the sponsor.

The protocol and the informed consent form were approved by the appropriate ethics committee and institutional review board associated with each study site. The study was conducted in accordance with Good Clinical Practice guidelines according to the International Council on Harmonisation Harmonised Tripartite Guideline and all local regulations. All patients provided written informed consent before study participation.

\subsection{Patients}

Patients had to meet the following criteria for inclusion in PB-06001 [10]: aged $\geq 18$ years with GD, naïve to ERT or no ERT in the past 12 months, and negative anti-glucocerebrosidase antibody test; splenomegaly, defined as spleen volume $\geq 8$ times the normal volume expected based on body weight; and thrombocytopenia, defined as a platelet count $<120,000 / \mathrm{mm}^{3}$ with or without anemia. Exclusion criteria for PB-06-001 included presence of signs and symptoms characteristic of neuronopathic GD and previous anaphylactoid reaction to alglucerase or imiglucerase and use of another experimental drug (for any disease) and presence of any medical, emotional, behavioral, or psychological condition that in the judgment of the investigator would interfere with the patient's compliance with the requirements of the study. Patients had to have completed PB-01-001 and PB-06-003 to be included in PB-06-007.
Exclusion criteria for PB-06-007 included current use of another investigational drug for any condition and presence of any medical, emotional, behavioral, or psychological condition that, in the judgment of the investigator, would interfere with the patient's compliance with the requirements of the study.

\subsection{Treatment}

For all patients, taliglucerase alfa was administered intravenously at 2-week intervals at the same dose as the last treatment in PB-06-003; dose adjustments were allowed.

\subsection{Efficacy assessments}

Spleen and liver volume were measured by volumetric magnetic resonance imaging using a validated system and blinded central readers, as described previously $[10,13]$. Volumes were expressed as multiples of normal (MN), where normal spleen volume is $2 \mathrm{~mL} / \mathrm{kg}$ of body weight $(\mathrm{kg}$ ) and normal liver volume is $25 \mathrm{~mL} / \mathrm{kg}$ of body weight (kg). Hemoglobin concentration and platelet count were measured along with the biomarkers of chitotriosidase activity and chemokine (C-C motif) ligand 18 (CCL18) concentration. Due to assay variability and for biomarker measurement consistency, the baseline samples obtained during Study PB-06-001 were re-run with the on-treatment samples for each time point from studies PB-06-001, PB-06-003, and PB-06-007 for chitotriosidase activity and CCL18 concentration; patients who discontinued the study were excluded entirely from biomarker analyses.

Measurement of bone marrow fat fraction was performed as an exploratory endpoint in a subset of the patients who underwent quantitative chemical shift imaging (QCSI) of the lumbar spine, as previously described [14]. The QCSI measurements were performed during Study PB-06-007 at 48 and 60 total months of taliglucerase alfa treatment. Measurements were compared with data from a Dutch GD population analysis, which demonstrated that mean lumbar spine fat fraction in healthy persons is approximately 0.37 , whereas a fat fraction of $\leq 0.23$ is associated with increased risk of bone complications [15].

\subsection{Safety assessments}

Safety assessments included the incidence and severity of AEs, physical examination findings, clinical laboratory values, electrocardiogram (ECG), echocardiogram, and pulmonary function tests. Anti-taliglucerase alfa antibodies and, in patients who had a positive antibody response, neutralizing antibodies were assessed using a validated analytical method.

\subsection{Statistical analysis}

For each parameter, efficacy analyses included those patients for whom data were available at each time point. The baseline for efficacy data was that for the beginning of the original taliglucerase alfa study (PB-06-001), and time points were counted continuously through PB06-007. Change and percent change from baseline were summarized for spleen volume, liver volume, hemoglobin, platelet counts, and biomarkers (chitotriosidase and/or CCL18) using descriptive statistics.

Descriptive statistics for continuous variables, sample size, mean and standard error (SE) of the mean (efficacy analysis parameters), standard deviation (summary demographic parameters), median, and range were calculated. For categorical variables, count and percentage are presented. No inferential statistical analyses were performed for testing the change from baseline and/or for comparisons between or among treatment groups. Data from 2 patients whose dose was adjusted were analyzed separately. 


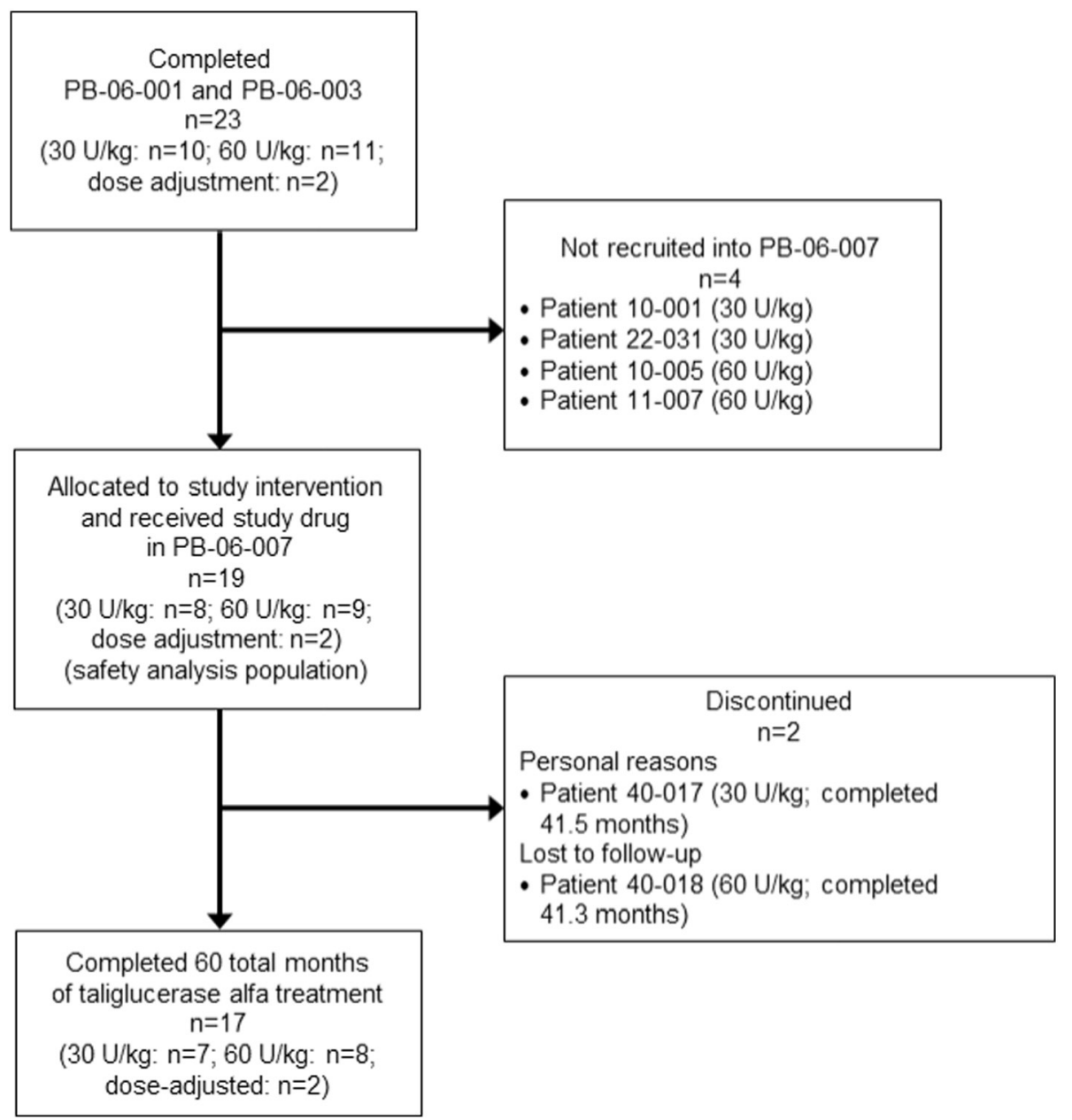

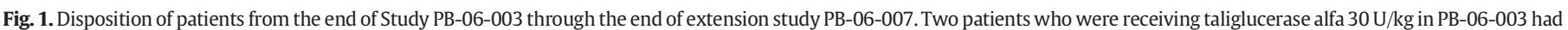

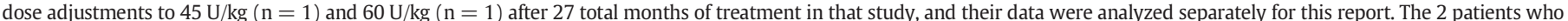
discontinued did so prior to any efficacy measurements in Study PB-06-007.

The safety population included all patients who received at least 1 dose of study drug in PB-06-007. AEs reported here are those that occurred during PB-06-007.

\section{Results}

Patient disposition is shown in Fig. 1. A total of 19 patients from 9 study sites enrolled in PB-06-007; 8 patients continued receiving taliglucerase alfa $30 \mathrm{U} / \mathrm{kg}$, 9 patients continued receiving taliglucerase alfa $60 \mathrm{U} / \mathrm{kg}$, and 2 patients were receiving taliglucerase alfa $30 \mathrm{U} / \mathrm{kg}$ in PB-06-003 and had dose adjustments to $45 \mathrm{U} / \mathrm{kg}(11-014)$ and $60 \mathrm{U} / \mathrm{kg}$ (42-025) in that study after 27 total months of treatment. Data for these 2 patients with dose adjustments were analyzed separately for this report. A total of 17 patients completed PB-06-007, and each received taliglucerase alfa infusions for a total of 60 months ( 5 years). Two patients discontinued from the study: One patient (40$017 ; 30 \mathrm{U} / \mathrm{kg}$ ) discontinued for personal logistical reasons after receiving infusions in Study PB-06-007 (41.5 total months of treatment); the other patient (40-018; $60 \mathrm{U} / \mathrm{kg}$ ) was lost to follow-up after receiving 4 infusions in Study PB-06-007 (41.3 total months of treatment). The patients discontinued prior to any efficacy measurements in PB-06007; their efficacy in Study PB-06-003 is reported elsewhere [11,12]. Baseline patient demographics are shown in Table 1, and baseline disease characteristics are shown in Table 2. Individual patient demographics, dose, duration of treatment, and baseline disease parameters are listed in Supplementary Tables S1-3.

Table 1

Baseline patient demographics.

\begin{tabular}{|c|c|c|c|}
\hline & $\begin{array}{l}\text { Taliglucerase alfa } \\
30 \mathrm{U} / \mathrm{kg}\end{array}$ & $\begin{array}{l}\text { Taliglucerase alfa } \\
60 \mathrm{U} / \mathrm{kg}\end{array}$ & Dose adjustment \\
\hline & $(\mathrm{n}=8)$ & $(\mathrm{n}=9)$ & $(n=2)$ \\
\hline \multicolumn{4}{|l|}{ Age, years } \\
\hline Mean (SD) & $44.8(13.4)$ & $39.9(12.0)$ & $38.5(0.7)$ \\
\hline Median (range) & $40.5(36-77)$ & $37.0(23-57)$ & a \\
\hline \multicolumn{4}{|l|}{ Sex, n (\%) } \\
\hline Male & $4(50.0)$ & $6(66.7)$ & $1(50.0)$ \\
\hline Female & $4(50.0)$ & $3(33.3)$ & $1(50.0)$ \\
\hline \multicolumn{4}{|l|}{ Race, n (\%) } \\
\hline Caucasian & $8(100.0)$ & $9(100.0)$ & $2(100.0)$ \\
\hline Other & $0(0.0)$ & $0(0.0)$ & $0(0.0)$ \\
\hline \multicolumn{4}{|l|}{ Ethnicity, n (\%) } \\
\hline Jewish-Ashkenazi & $2(25.0)$ & $1(11.1)$ & $1(50.0)$ \\
\hline Non-Jewish & $6(75.0)$ & $8(88.9)$ & $1(50.0)$ \\
\hline
\end{tabular}

SD, standard deviation.

a Median not applicable for $\mathrm{n}=2$. 
Table 2

Baseline ${ }^{\text {a }}$ disease characteristics.

\begin{tabular}{|c|c|c|c|}
\hline & Taliglucerase alfa $30 \mathrm{U} / \mathrm{kg}$ & Taliglucerase alfa $60 \mathrm{U} / \mathrm{kg}$ & Dose adjustment \\
\hline & $(\mathrm{n}=8)$ & $(n=9)$ & $(\mathrm{n}=2)$ \\
\hline \multicolumn{4}{|l|}{ Spleen volume, MN } \\
\hline Mean (SE) & $14.5(3.1)$ & $15.8(4.9)$ & $19.8(8.2)$ \\
\hline Median (range) & $12.1\left(7.7^{b}-34.5\right)$ & $10.7\left(7.8^{\mathrm{b}}-54.2\right)$ & $(11.6 ; 28.0)^{\mathrm{c}}$ \\
\hline \multicolumn{4}{|l|}{ Liver volume, MN } \\
\hline Mean (SE) & $1.8(0.2)$ & $1.6(0.2)$ & $1.6(0.1)$ \\
\hline Median (range) & $1.7(1.4-2.9)$ & $1.4(0.9-2.6)$ & $(1.5 ; 1.7)^{\mathrm{c}}$ \\
\hline \multicolumn{4}{|l|}{ Hemoglobin, g/dL } \\
\hline Mean (SE) & $12.3(0.7)$ & $11.6(0.9)$ & $12.5(0.9)$ \\
\hline Median (range) & $13.0(7.9-14.6)$ & $10.3(9.0-16.0)$ & $(11.6 ; 13.4)^{\mathrm{C}}$ \\
\hline \multicolumn{4}{|c|}{ Platelet counts, $/ \mathrm{mm}^{3}$} \\
\hline Mean (SE) & $70,975(8700.6)$ & $70,067(7695.5)$ & $69,500(42,500)$ \\
\hline Median (range) & $70,500(42,800-107,000)$ & $70,000(40,000-103,000)$ & $(27,000 ; 112,000)^{\mathrm{c}}$ \\
\hline \multicolumn{4}{|c|}{ Chitotriosidase activity ${ }^{\mathrm{d}}, \mathrm{nmol} / \mathrm{mg} \cdot \mathrm{h}$} \\
\hline Mean (SE) & $27,632(4654.9)$ & $21,023(8110.4)$ & $28,102(1763.5)$ \\
\hline Median (range) & $33,161(7631-41,201)$ & $15,169(3035-62,351)$ & $(26,338 ; 29,865)^{\mathrm{c}}$ \\
\hline \multicolumn{4}{|l|}{$\mathrm{CCL} 18, \mathrm{ng} / \mathrm{mL}^{\mathrm{e}}$} \\
\hline Mean (SE) & $793.7(94.8)$ & 899.9 (162.8) & 1011.5 (373.5) \\
\hline Median (range) & $810.0(479-1248)$ & $799.0(489-1882)$ & $(638 ; 1385)^{\mathrm{c}}$ \\
\hline
\end{tabular}

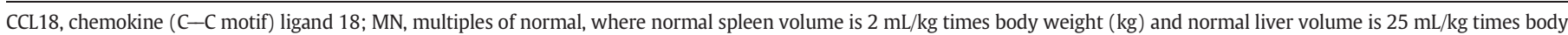
weight $(\mathrm{kg})$; SE, standard error.

a Baseline values are from the original study (PB-06-001).

b Volume rounded up to 8.0 for Study PB-06-001 inclusion purposes.

c Median not applicable for $\mathrm{n}=2$.

d Taliglucerase alfa $30 \mathrm{U} / \mathrm{kg}$ and $60 \mathrm{U} / \mathrm{kg}$ groups, $\mathrm{n}=7$ for each.

e Taliglucerase alfa $30 \mathrm{U} / \mathrm{kg}, \mathrm{n}=7$.

\subsection{Efficacy}

Individual patient efficacy results are listed in Supplementary Tables S1-3. Mean spleen and liver volumes showed continuous decreases during 60 months of treatment with taliglucerase alfa $30 \mathrm{U} / \mathrm{kg}$ and $60 \mathrm{U} / \mathrm{kg}$ (Fig. 2). Mean reductions (SE) in spleen volume for the 16 patients who completed 60 months and had organ volume measurements were $-8.7 \mathrm{MN}(2.0 \mathrm{MN} ; \mathrm{n}=7)$ for patients receiving $30 \mathrm{U} / \mathrm{kg}$, $-6.9 \mathrm{MN}(0.8 \mathrm{MN} ; \mathrm{n}=7$ ) for patients receiving $60 \mathrm{U} / \mathrm{kg}$, and $-12.4 \mathrm{MN}$ (5.8 MN; $\mathrm{n}=2$ ) for patients who had a dose adjustment; these represented mean percent reductions (SE) of $-56.7 \%$ (3.7\%), $-67.9 \%$ (2.9\%), and $-61.0 \%$ (4.3\%) for these groups, respectively. Mean reductions from baseline to 60 months in liver volumes were $-0.6 \mathrm{MN}(0.1 \mathrm{MN} ; \mathrm{n}=7)$ for patients receiving $30 \mathrm{U} / \mathrm{kg},-0.4 \mathrm{MN}$ $(0.1 \mathrm{MN} ; \mathrm{n}=7$ ) for patients receiving $60 \mathrm{U} / \mathrm{kg}$, and $-0.5 \mathrm{MN}$ $(0.1 \mathrm{MN} ; \mathrm{n}=2)$ for patients who had a dose adjustment; these represented mean percent reductions (SE) of $-32.5 \%$ (1.9\%), $-23.3 \%$ (7.7\%), and $-30.4 \%(2.4 \%)$, respectively.

Mean hemoglobin concentration rose through the first 12 months and remained stable over the ensuing 48 months of therapy with taliglucerase alfa therapy (Fig. 3). Mean changes (SE) from baseline to 60 months in hemoglobin concentration were $+14.1 \mathrm{mg} / \mathrm{dL}$ $(0.9 \mathrm{mg} / \mathrm{dL} ; \mathrm{n}=7)$ for patients receiving $30 \mathrm{U} / \mathrm{kg},+13.9 \mathrm{mg} / \mathrm{dL}$ $(0.6 \mathrm{mg} / \mathrm{dL} ; \mathrm{n}=8)$ for patients receiving $60 \mathrm{U} / \mathrm{kg}$, and $+14.3 \mathrm{mg} / \mathrm{dL}$ $(0.1 \mathrm{mg} / \mathrm{dL} ; \mathrm{n}=2)$ for patients who had a dose adjustment; these represented mean percent changes (SE) of $+18.8 \%(4.8 \%),+20.5 \%(5.9 \%)$, and $+14.6 \%(7.8 \%)$ for these groups, respectively. Platelet counts rose continuously in parallel with the continuous improvement in spleen volume. Mean increases from baseline to 60 months in platelet count were $+31,871 / \mathrm{mm}^{3}\left(14,299 / \mathrm{mm}^{3} ; \mathrm{n}=7\right)$ for patients receiving $30 \mathrm{U} / \mathrm{kg},+106,800 / \mathrm{mm}^{3}\left(18,967 / \mathrm{mm}^{3} ; \mathrm{n}=8\right)$ for patients receiving $60 \mathrm{U} / \mathrm{kg}$, and $+34,000 / \mathrm{mm}^{3}\left(18,000 / \mathrm{mm}^{3} ; \mathrm{n}=2\right)$ for patients who had a dose adjustment; these represented mean percent changes (SE) of $+44.8 \%(20.5 \%),+168.0 \%$ (41.4\%), and $+52.8 \%$ (6.4\%), respectively.

Chitotriosidase activity and CCL18 concentration decreased during the 60 months of treatment with taliglucerase alfa (Fig. 4). At month 60 , chitotriosidase activity decreased from baseline by a mean (SE) of $-83.1 \%(4.7 \% ; n=7$ ) for patients receiving $30 \mathrm{U} / \mathrm{kg}$,
$-93.4 \%(1.7 \% ; \mathrm{n}=7)$ for patients receiving $60 \mathrm{U} / \mathrm{kg}$, and $-87.9 \%$ $(5.0 \% ; \mathrm{n}=2)$ for patients who had a dose adjustment; mean percent decreases from baseline to 60 months in CCL18 concentration were $-66.7 \%(4.7 \% ; n=7),-83.3 \%$ (3.3\%; $n=8)$, and $-78.9 \%$ (6.7\%; $\mathrm{n}=2$ ), respectively.

Lumbar spine fat fraction was measured at months 48 and 60 for 5 patients (3 receiving $30 \mathrm{U} / \mathrm{kg}$ and 2 receiving $60 \mathrm{U} / \mathrm{kg}$ ) using QCSI [14, 15]. All 5 patients demonstrated increases in lumbar spine fat fraction from baseline (range $0.13-0.32$ ). At baseline, 1 patient had a fat fraction $>0.23$, which increased to 0.46 by month 60 . Four patients had lumbar spine fat fraction $\leq 0.23$ at baseline; by month 48,3 of these patients had values $\geq 0.37$, which was maintained through 60 total months of treatment. The 1 patient who did not achieve a fat fraction $\geq 0.37$ had the lowest baseline value (0.11) and improved to 0.29 at month 60 .

\subsection{Safety}

Taliglucerase alfa was well tolerated. AEs occurring during Study PB06-007, based on all enrolled patients $(N=19)$, are summarized in Table 3. The most common AEs were nasopharyngitis $(26.3 \% ; n=5)$ and arthralgia ( $15.8 \% ; n=3$ ). The majority of AEs were mild to moderate in intensity. Two severe non-treatment-related AEs were reported in 2 patients receiving taliglucerase alfa $60 \mathrm{U} / \mathrm{kg}$ : a very severe (Grade 4) right hip arthrosis that required surgical artificial hip implantation (30-011) and a severe (Grade 3) papillary thyroid carcinoma that necessitated a total thyroidectomy and left node resection (42-026). Both of these events were also considered serious AEs; no other serious AEs were reported. None of the AEs reported during PB-06-007 were considered to be related to treatment. No patient experienced anaphylaxis during Study PB-06-007. One patient (30-009) who experienced hypersensitivity during $\mathrm{PB}-06-003$ did not receive pre-medication and did not have a recurrence during PB-06-007.

Most laboratory test values remained normal through study end. No abnormal values were clinically meaningful or reported as AEs during the study, with the exception of those for 2 patients (14-027 and 15016), which were not considered to be related to treatment. No abnormal findings were observed in any of the ECG parameters, and no clinically significant changes in ECG findings were noted during the study. 
A

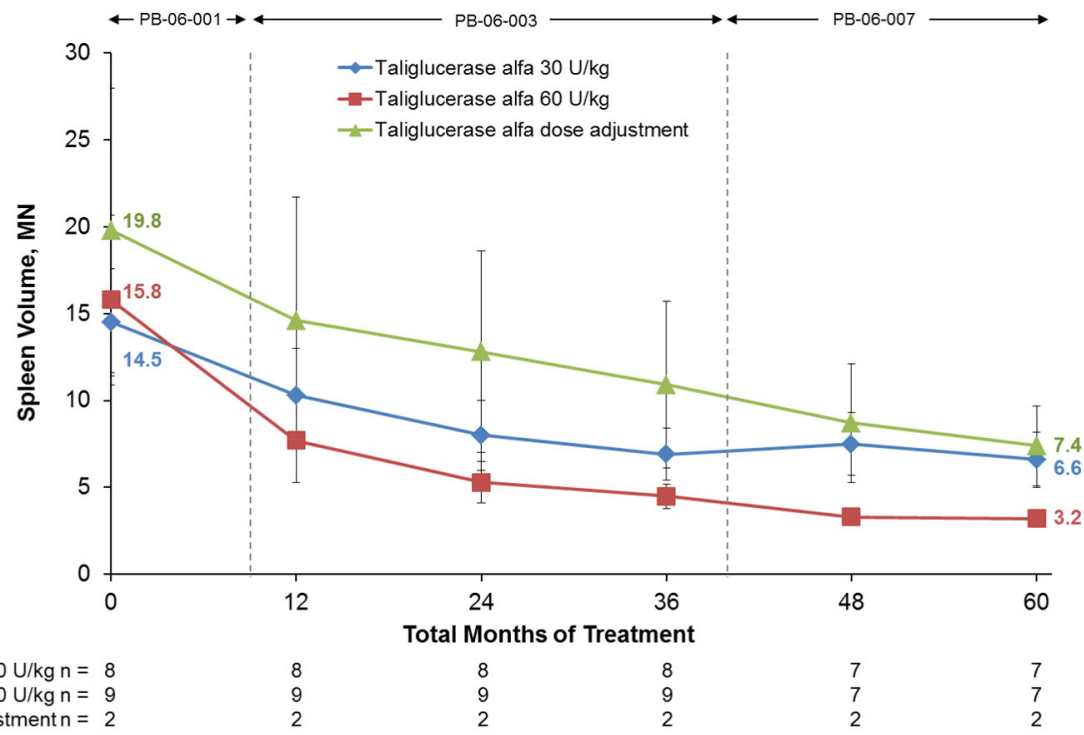

B

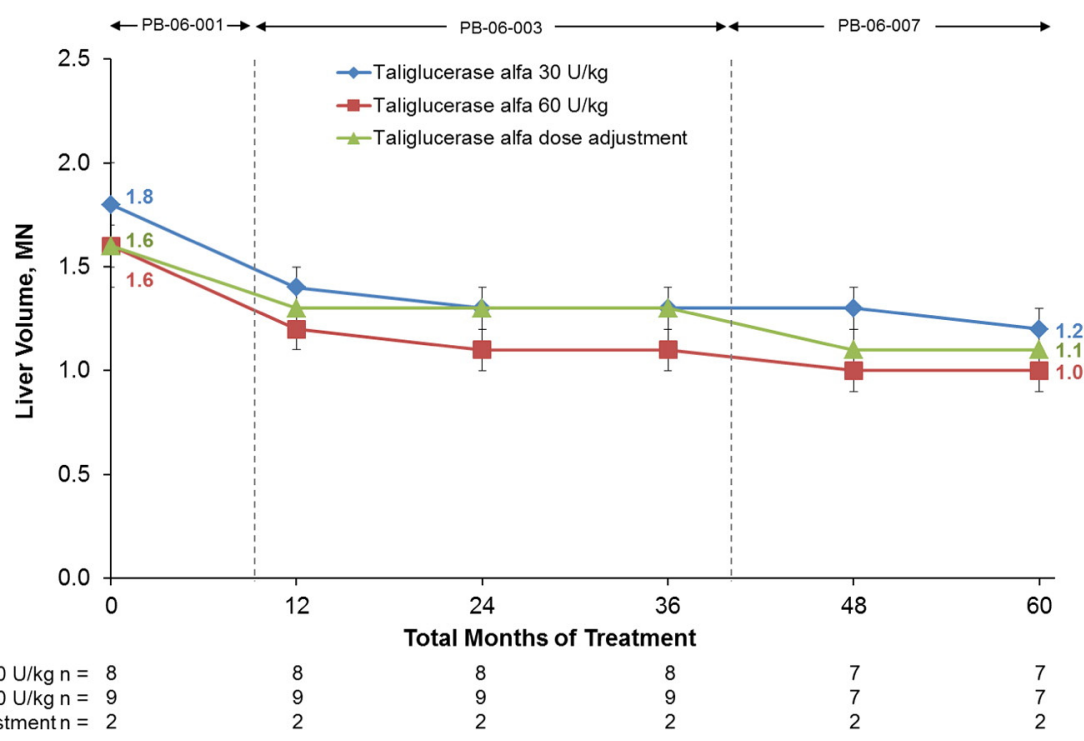

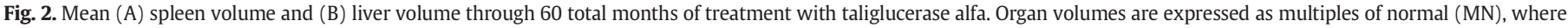

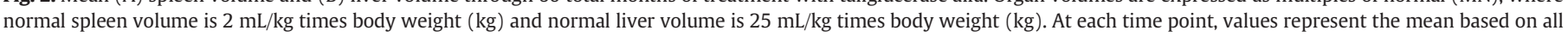

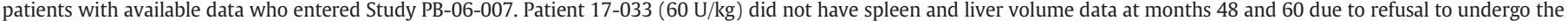

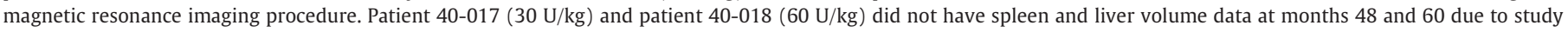

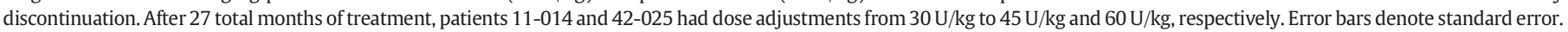

Two patients (17-032 and 15-016) had abnormal echocardiography results; these consisted of left ventricular dysfunction at month 60 in 1 patient and fusion of the right and left coronary cusps, first reported during PB-06-003 and continuing at month 48 . Both patients continued to receive taliglucerase alfa for 60 months and completed the study.

A total of 6 patients reported bone pain during Study PB-06-007. Reports include spine pain (patient 30-008; $30 \mathrm{U} / \mathrm{kg}$ ), lower back pain (patient 41-022; $30 \mathrm{U} / \mathrm{kg}$ ), left knee pain (patient 30-009; $60 \mathrm{U} / \mathrm{kg}$ ), right knee pain, left hip pain, and generalized pain in pelvic bones (patient 30-011; $60 \mathrm{U} / \mathrm{kg}$ ), right knee pain (patient 42-026; $60 \mathrm{U} / \mathrm{kg}$ ), and intermittent bone pain in the left shin (patient 11-014; dose-adjustment group). None of these events were reported as treatment-related.

\subsection{Antidrug antibodies}

Of the patients who entered PB-06-007, 8 had previously tested positive for immunoglobulin $\mathrm{G}(\operatorname{IgG})$ antibodies to taliglucerase alfa (Supplementary Tables S1 and S2). In PB-06-007, 5 patients tested positive for IgG antibodies to taliglucerase alfa; all were among those who previously tested positive in PB-06-003. Neither of the patients who discontinued Study PB-06-007 tested positive for IgG antibodies to taliglucerase alfa at any time during all 3 studies. Of the 5 patients who tested positive for $\operatorname{IgG}$ antibodies to taliglucerase alfa in PB-06-007, 3 (10-028, 15-016, and 30-008) tested positive and 2 (17-032 and 30-009) tested negative for neutralizing activity based on an in vitro enzymatic activity assay in PB06-007. No new treatment-related AEs were reported in patients who tested positive for anti-taliglucerase alfa IgG antibodies in PB-06-007. 

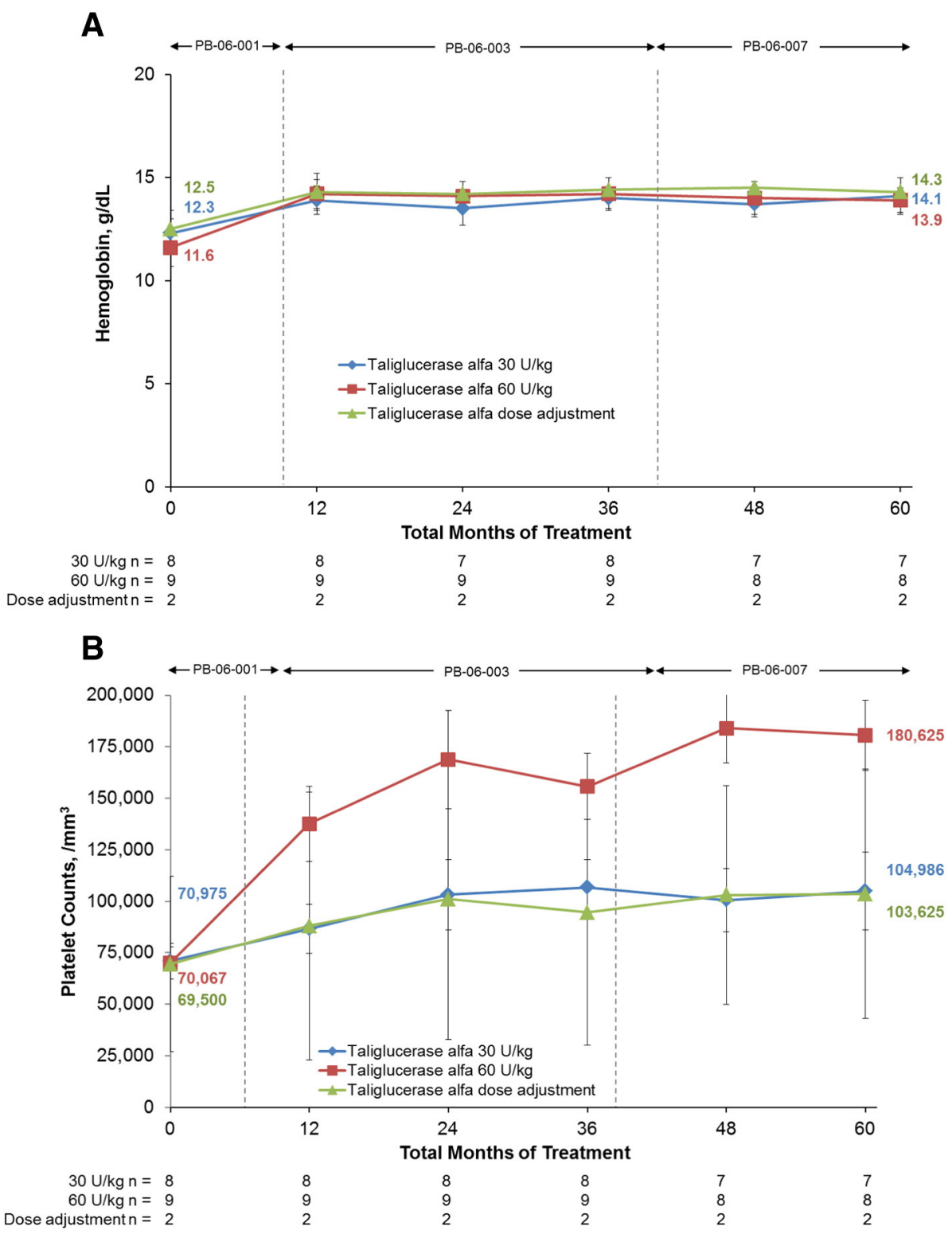

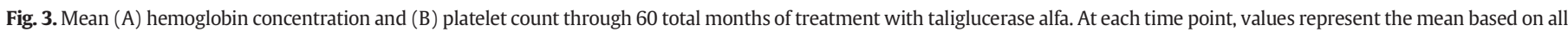

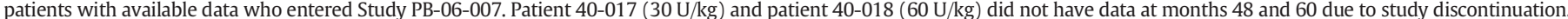
After 27 total months of treatment, patients 11-014 and 42-025 had dose adjustments from $30 \mathrm{U} / \mathrm{kg}$ to $45 \mathrm{U} / \mathrm{kg}$ and $60 \mathrm{U} / \mathrm{kg}$, respectively. Error bars denote standard error.

\section{Discussion}

Taliglucerase alfa, an ERT approved for treatment of GD, is the first FDA-approved plant cell-expressed recombinant therapeutic protein. Study PB-06-007 was a long-term treatment follow-up of the multicenter, multinational, 2-dose (30 U/kg and $60 \mathrm{U} / \mathrm{kg}$ ) taliglucerase alfa phase 3 pivotal study, PB-06-001, and its extension study PB-06-003, representing the final 21 months of the total 60 months of taliglucerase alfa in (initially) treatment-naïve adults with GD. Spleen volume, liver volume, chitotriosidase activity, and CCL18 concentration decreased continuously over the 60 months of taliglucerase alfa treatment. Platelet counts increased continuously over the 60 months of treatment, and hemoglobin concentration rose during the first 12 months of therapy and remained stable through the end of the study. Treatment was well tolerated, with mostly mild to moderate AEs and no AEs considered related to treatment.

QCSI measurements of lumbar spine fat fraction in 5 patients showed that all experienced improvement in this exploratory endpoint.
Comparison to data from a Dutch QCSI population study [15] indicated that 4 patients were at risk for bone complications $(\leq 0.23)$ at baseline. Three of these patients achieved levels found to be normal in the Dutch population $(\geq 0.37)$ by 48 months of taliglucerase alfa treatment, which was maintained through 60 total months of treatment. One patient who did not achieve a level of $\geq 0.37$ by 60 months had the lowest baseline measurement and reached a level of 0.29 by 60 months, which was considered to be above the level for risk of bone complications. Thus, this exploratory analysis of lumbar spine fat fraction by QCSI of 5 patients indicated that taliglucerase alfa treatment resulted in clinically meaningful improvements.

The limitations of this study include the small number of patients in each dose group, which is common for studies involving rare diseases such as GD. While the patients in the $60 \mathrm{U} / \mathrm{kg}$ dose group appeared to achieve more rapid and profound responses, these observations should take the small sample size into consideration.

The significance of the anti-taliglucerase alfa antibody findings is unclear at this time. In patients with positive results for anti-taliglucerase 
A

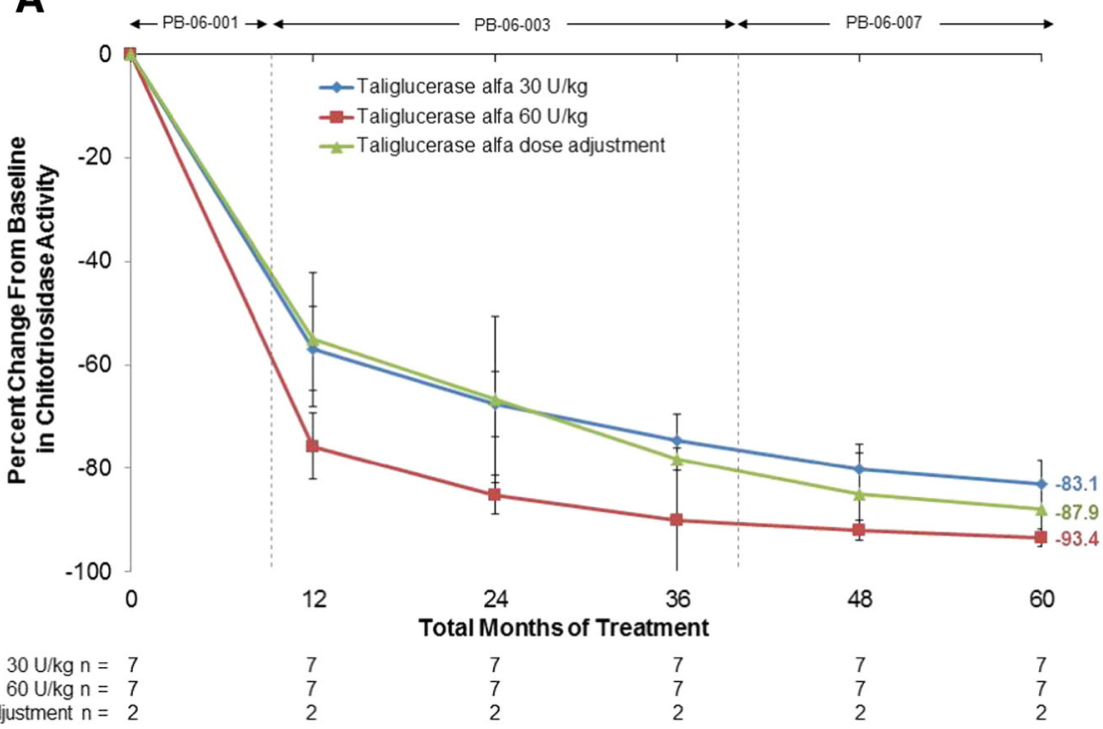

Dose adjustment $n=2$

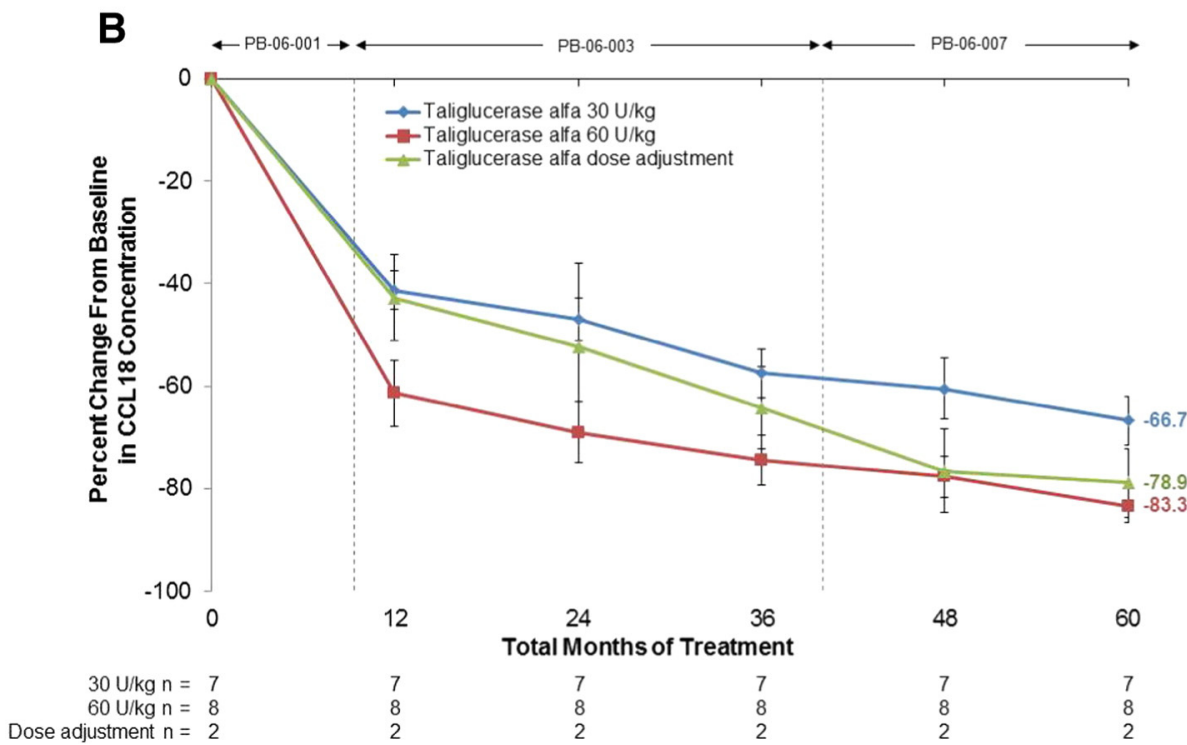

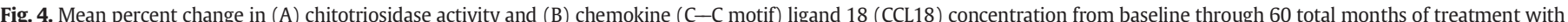

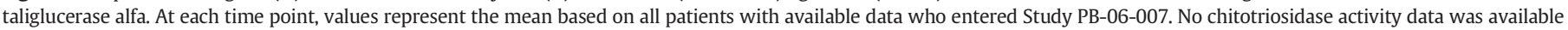

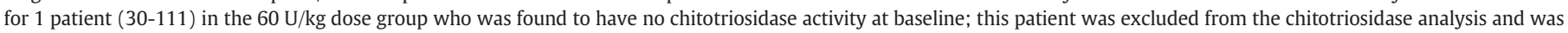

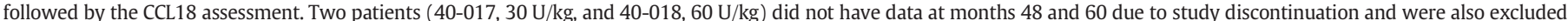

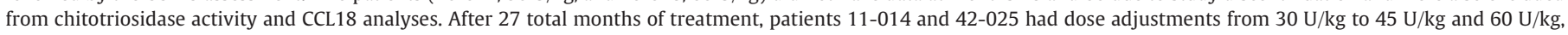
respectively. Error bars denote standard error.

alfa IgG or neutralizing antibody assay results, there was no effect on the

Table 3

Adverse events occurring in Study PB-06-007 ( $\mathrm{N}=19)$.

\begin{tabular}{lll}
\hline & $\begin{array}{l}\text { No. of adverse events } \\
\text { (no. of patients) }\end{array}$ & Percentage of total adverse events \\
\hline Total adverse events & $73(15)$ & 100.0 \\
Mild or moderate & $71(15)$ & 97.3 \\
Severe/very severe & $2(2)$ & 2.7 \\
$30 \mathrm{U} / \mathrm{kg}$ & $0(0)$ & 0.0 \\
$60 \mathrm{U} / \mathrm{kg}$ & $2(2)$ & 0.0 \\
Serious & $2(2)$ & 2.7 \\
Treatment-related $^{\mathrm{b}}$ & $0(0)$ & 0.0 \\
\hline
\end{tabular}

a Adverse events occurring in extension study PB-06-007 and based on all patients enrolled in that study $(\mathrm{N}=19)$.

b Right hip arthrosis requiring artificial hip replacement (patient 30-011) and papillary thyroid carcinoma requiring thyroidectomy (patient 42-026). efficacy parameters of spleen volume, liver volume, hemoglobin concentration, platelet count, or biomarkers based on evaluations of these parameters from the original study from baseline to last observation, although 1 patient with anti-taliglucerase alfa antibodies appeared to experience a decrease in platelet count $(30-008 ; 30 \mathrm{U} / \mathrm{kg}$; decrease from $86,000 / \mathrm{mm}^{3}$ to $64,000 / \mathrm{mm}^{3}$ ), and another patient experienced a small increase in liver volume (14-027; $60 \mathrm{U} / \mathrm{kg}$; increase from $1.3 \mathrm{MN}$ to $1.4 \mathrm{MN}$ ); however, improvements were observed for all other efficacy parameters for both of these patients. It should be noted that comparison of the incidence of taliglucerase alfa antibodies to that of other products can be misleading and should be approached with caution because assay results are highly dependent on the sensitivity and specificity of each assay and may be influenced by several factors, including assay methodology, sample handling, timing of sample collection, concomitant medication, and underlying disease [8]. 


\section{Conclusions}

Long-term improvements in visceral and hematologic disease parameters and in biomarkers were observed in 17 treatment-naïve adult patients with GD during 5 years of treatment with taliglucerase alfa. These findings represent long-term continuous evaluation of taliglucerase alfa in treatment-naïve patients with GD and extend the clinical efficacy and safety dataset for taliglucerase alfa at 2 dose levels over a 5-year period. In this study, taliglucerase alfa was well tolerated, and no new safety concerns emerged.

\section{Disclosures}

A.Z. was a consultant for Protalix BioTherapeutics during the course of the clinical trials of taliglucerase alfa; is a member of the speakers bureau for Genzyme/Sanofi, Pfizer, and Shire; and has received honoraria from Genzyme/Sanofi, Pfizer, and Shire. In addition, the Gaucher Clinic receives grant/research support from Genzyme/Sanofi and Shire. G.D., P.G., H.R., F.G., M.P., E.T.M., S.E.S.-M., P.A.C., and S.V. were study investigators. R.C. and S.A. are employees of Protalix BioTherapeutics.

\section{Author contributions}

None of the authors received compensation for their contributions to this manuscript. All authors contributed to data interpretation, reviewed and provided their comments on this manuscript, and approved the final version.

\section{Acknowledgments}

This study was sponsored by Protalix BioTherapeutics. Editorial and medical writing support was provided by Elizabeth Daro-Kaftan, PhD, of Peloton Advantage, LLC, and was funded by Pfizer. Pfizer and Protalix BioTherapeutics entered into an agreement in November 2009 to develop and commercialize taliglucerase alfa.

\section{Appendix A. Supplementary data}

Supplementary data to this article can be found online at http://dx. doi.org/10.1016/j.bcmd.2016.07.002.

\section{References}

[1] A. Zimran, D. Elstein, Gaucher disease and related lysosomal storage diseases, in: K Kaushansky, M.A. Lichtman, J.T. Prchal, M.M. Levi, O. Press, L. Burns, M. Caligiuri (Eds.), Williams Hematology, McGraw-Hill Education, New York, NY 2016, pp. 1121-1133.

[2] G.A. Grabowski, E.H. Kolodny, N.J. Weinreb, et al., Gaucher disease: phenotypic and genetic variation, in: D. Valle, A.L. Beaudet, B. Vogelstein, K.W. Kinzler, S.E. Antonarakis, A. Ballabio, C.R. Scriver, W.S. Sly, F. Bunz, K.M. Gibson, G. Mitchell (Eds.), The Online Metabolic and Molecular Bases of Inherited Disease, McGraw Hill Companies, New York, NY 2010, pp. 1-63.

[3] J. Charrow, H.C. Andersson, P. Kaplan, et al., The Gaucher registry: demographics and disease characteristics of 1698 patients with Gaucher disease, Arch. Intern. Med. 160 (2000) 2835-2843.

[4] P.K. Mistry, N. Belmatoug, S. vom Dahl, R. Giugliani, Understanding the natural history of Gaucher disease, Am. J. Hematol. 90 (Suppl. 1) (2015) S6-S11.

[5] N.W. Barton, R.O. Brady, J.M. Dambrosia, et al., Replacement therapy for inherited enzyme deficiency-macrophage-targeted glucocerebrosidase for Gaucher's disease, N. Engl. J. Med. 324 (1991) 1464-1470.

[6] A. Zimran, D. Elstein, Management of Gaucher disease: enzyme replacement therapy, Pediatr. Endocrinol. Rev. 12 (Suppl. 1) (2014) 82-87.

[7] J.L. Fox, First plant-made biologic approved, Nat. Biotechnol. 30 (2012) 472.

[8] Elelyso [Package Insert], Pfizer Labs, New York, NY, 2015.

[9] Y. Shaaltiel, D. Bartfeld, S. Hashmueli, et al., Production of glucocerebrosidase with terminal mannose glycans for enzyme replacement therapy of Gaucher's disease using a plant cell system, Plant Biotechnol. J. 5 (2007) 579-590.

[10] A. Zimran, E. Brill-Almon, R. Chertkoff, et al., Pivotal trial with plant cell-expressed recombinant glucocerebrosidase, taliglucerase alfa, a novel enzyme replacement therapy for Gaucher disease, Blood 118 (2011) 5767-5773.

[11] A. Zimran, G. Duran, A. Mehta, et al., Long-term efficacy and safety results of taliglucerase alfa up to 36 months in adult treatment-naive patients with Gaucher disease, Am. J. Hematol. 91 (2016) 656-660.

[12] G.M. Pastores, S.P. Shankar, M. Petakov, et al., Enzyme replacement therapy with taliglucerase alfa: 36-month safety and efficacy results in adult patients with Gaucher disease previously treated with imiglucerase, Am. J. Hematol. 91 (2016) 661-665.

[13] L. Bracoud, H. Ahmad, E. Brill-Almon, R. Chertkoff, Improving the accuracy of MRI spleen and liver volume measurements: a phase III Gaucher disease clinical trial setting as a model, Blood Cells Mol. Dis. 46 (2011) 47-52.

[14] L. van Dussen, A. Zimran, E.M. Akkerman, et al., Taliglucerase alfa leads to favorable bone marrow responses in patients with type I Gaucher disease, Blood Cells Mol. Dis. 50 (2013) 206-211.

[15] M. Maas, C.E. Hollak, E.M. Akkerman, et al., Quantification of skeletal involvement in adults with type I Gaucher's disease: fat fraction measured by Dixon quantitative chemical shift imaging as a valid parameter, AJR Am. J. Roentgenol. 179 (2002) 961-965. 